\title{
Lifelong Bilingualism Maintains White Matter Integrity in Older Adults
}

\author{
Gigi Luk, ${ }^{1,5}$ Ellen Bialystok, ${ }^{1,3}$ Fergus I. M. Craik, ${ }^{1,2}$ and Cheryl L. Grady ${ }^{1,2,4}$ \\ ${ }^{1}$ Rotman Research Institute at Baycrest, Toronto, Ontario M6A 2E1, Canada, ${ }^{2}$ Department of Psychology, University of Toronto, Toronto, Ontario M5S 3G3, \\ Canada, ${ }^{3}$ Department of Psychology, York University, Toronto, Ontario M3J 1P3, Canada, ${ }^{4}$ Department of Psychiatry, University of Toronto, Toronto, \\ Ontario M5T 1R8, Canada, and ${ }^{5}$ Harvard Graduate School of Education, Cambridge, Massachusetts 02138
}

Previous research has shown that bilingual speakers have higher levels of cognitive control than comparable monolinguals, especially at older ages. The present study investigates a possible neural correlate of this behavioral effect. Given that white matter (WM) integrity decreases with age in adulthood, we tested the hypothesis that bilingualism is associated with maintenance of WM in older people. Using diffusion tensor imaging, we found higher WM integrity in older people who were lifelong bilinguals than in monolinguals. This maintained integrity was measured by fractional anisotropy (FA) and was found in the corpus callosum extending to the superior and inferior longitudinal fasciculi. We also hypothesized that stronger WM connections would be associated with more widely distributed patterns of functional connectivity in bilinguals. We tested this by assessing the resting-state functional connectivity of frontal lobe regions adjacent to WM areas with group differences in FA. Bilinguals showed stronger anterior to posterior functional connectivity compared to monolinguals. These results are the first evidence that maintained WM integrity is related to lifelong naturally occurring experience; the resulting enhanced structural and functional connectivity may provide a neural basis for "brain reserve."

\section{Introduction}

The cognitive decline associated with older age can be influenced by language experience. Older bilinguals typically show better executive function (EF) performance than their monolingual peers (Bialystok et al., 2008; see Bialystok and Craik, 2010 for a review). The bilingual participants in these studies had used both languages actively on a daily basis since childhood, so had accumulated a considerable amount of experience in manipulating both languages. Despite accumulating behavioral evidence, the neural mechanism behind this cognitive advantage is unknown. One hypothesis is that bilinguals recruit more distributed brain networks to manage two language systems and prolonged practice engages these neural systems to process information more efficiently, even when the task does not involve language. Neuroimaging studies with young bilinguals have indeed shown that cognitive performance on nonverbal executive function tasks is associated with utilization of more distributed brain networks than those used by monolinguals (Garbin et al., 2010; Luk et al., 2010; Moreno et al., 2010). The engagement of more distributed brain networks may reflect stronger white matter (WM) connec-

\footnotetext{
Received Sept. 7, 2011; revised; accepted Sept. 30, 2011.

Author contributions: G.L. and C.L.G. designed research; G.L. performed research; G.L. and C.L.G. analyzed data; G.L., E.B., F.I.C., and C.L.G. wrote the paper.

This work was supported by the Canadian Institutes of Health Research (MOP14036 to C.L.G.), the Canada Research Chairs program, the Ontario Research Fund, the Canadian Foundation for Innovation, and the Heart and Stroke Foundation Centre for Stroke Recovery. We thank Annette Weeks-Holder and staff of the Baycrest fMRI centre for technical assistance.

The authors declare no competing financial interests.

Correspondence should be addressed to Gigi Luk, Harvard Graduate School of Education, 14 Appian Way, Larsen 506, Cambridge, MA 02138. E-mail: gigi_luk@gse.harvard.edu.

DOI:10.1523/JNEUROSCI.4563-11.2011

Copyright $\odot 2011$ the authors $\quad 0270-6474 / 11 / 3116808-06 \$ 15.00 / 0$
}

tivity between brain regions, facilitating information transfer and resulting in better executive performance. In older bilinguals, the decrease in white matter integrity (Madden et al., 2009) and reductions of gray matter volume (Fjell and Walhovd, 2010) known to accompany aging may be mitigated by their language experience. That is, better-maintained WM in older bilinguals might serve as a structural mechanism for behavioral differences found in previous research.

Diffusion tensor imaging (DTI) provides indirect measures of WM integrity that indicate the degree of anisotropic water diffusion constrained by axons (fractional anisotropy, or FA), such that higher FA indicates better integrity. Given the more distributed and bilateral functional networks recruited by bilinguals, we expected that bilingualism in older adults would be associated with higher FA in WM tracts that connect disparate brain regions, in particular, the corpus callosum (CC). FA can also be decomposed to obtain an estimate of diffusivity parallel to WM tracts (axial diffusivity, AD) or perpendicular to WM tracts (radial diffusivity, RD, Beaulieu, 2009). Given that lower RD is associated with lower water diffusion perpendicular to WM tracts and is more sensitive than $\mathrm{AD}$ to age-related cognitive differences (Madden et al., 2009), we expected that the bilinguals would have lower RD than monolinguals in regions showing a difference in FA.

Better WM integrity might also be associated with stronger functional connectivity between brain regions. To assess possible functional correlates of better maintained WM in bilinguals, we conducted a resting-state functional connectivity analysis to measure intrinsic functional connectivity of gray matter regions near WM structures showing a group difference. The goal was to identify differences in functional connectivity that might reflect 
fundamental differences in function independent of any taskspecific effects of bilingualism. Consistent with previous research showing more distributed brain activity in bilinguals, we expected to find more distributed resting-state functional connectivity involving regions with greater WM connectivity in bilinguals.

\section{Materials and Methods}

\section{Participants}

Twenty-eight right-handed healthy older adults [mean $(M)$ age $=70.5$ years, $\mathrm{SD}=3$ years] participated in the study. Fourteen participants were monolingual speakers of English (7 males and 7 females) and 14 had lifelong bilingual experience ( 6 males and 8 females). Participants provided informed consent and underwent a behavioral and a scanning session. The two groups had comparable demographic backgrounds and neuropsychological performance but different language experience (see Notes for more information). Two additional monolingual males were excluded from the analyses due to incidental findings on their MRIs. All the procedures were approved by the Research Ethics Board of the Baycrest Centre in Toronto, Canada. Monolingual older adults reported English to be their only communicating language, whereas the bilingual older adults reported that they had used both English and another alphabetic language regularly since childhood (before age 11). The monolingual and bilingual older adults were matched on age and gender. All participants were active community members, reported no known psychiatric or health issues that may affect neurological health, no experience of concussion, and no contraindication with MR scanning. Twotailed $t$ tests showed no statistical significant difference between the monolinguals and bilinguals in age, years of education, and weekly hours spent using a computer $\left(t_{(24)}<2, \mathrm{~ns}\right)$.

\section{Neuropsychological tasks}

Participants underwent a $1 \mathrm{~h}$ behavioral testing session consisting of a battery of neuropsychological tasks before the scanning session.

Mini-Mental State Examination (short form, Folstein et al., 1975). A short form of the MMSE that has a maximum of 17 points was administered. The task was a global screening measure for general cognitive ability, including orientation to place, attention, language, and complex commands.

Shipley Institute of Living Scale —Vocabulary test (Zachary, 1986). Participants were instructed to choose one of the four words that has a similar meaning to a target word. Forty trials were administered. Percentage of correct responses was computed.

Verbal fluency. Participants were asked to produce words that either begin with a specific letter (F, A, and S) or belong to a semantic category (clothing item, animals, and occupation) within $1 \mathrm{~min}$. Incorrect responses or repeated items were excluded from the calculation of the average number of correct responses.

Design fluency, Delis-Kaplan Executive Functions System (Delis et al., 2001). This subtest requires participants to create different designs using four straight lines to connect dots within $1 \mathrm{~min}$. In the baseline condition, only empty dots were shown. The second condition showed both empty and filled dots but the participants were instructed to connect empty dots only. Finally, in the switching condition, participants were asked to create designs by alternating the empty and filled dots. Incorrect responses or repeated items were excluded from the calculation of the average number of correct responses.

Stroop task (Stroop, 1935). Participants were asked to name the color of the ink in which the stimuli were printed. These colors were red, blue, pink, green, yellow, and black. Participants were asked to identify these colors before administering the task to ensure they had intact color vision. Three vertical columns with colored items were presented for each of the four conditions: baseline, interference, negative priming, and habituation. In the baseline condition, arrays of five X's printed in one of the six colors were presented as stimuli. In the interference condition, words that corresponded to the six colors were printed in a different color than the word indicated. In the negative priming condition, the word in any given trial corresponded to the color of the following trial, requiring the participants to name the color corresponding to the word suppressed in the previous trial. Finally, the habituation condition only had the word "blue" printed in one of the six colors. The time to finish naming the three columns was recorded.

Trail-making task (Reitan, 1958). Participants were asked to connect circles containing numbers or letters according to ascending numerical values, alphabetic order, and switching between numbers and letters. The numbers-only and switching conditions were from the standard battery (Trails A and B, respectively), and we included the letter-only condition as an additional baseline. Locations of the circles in the letter-only condition were the same as the number-only condition to control for the amount of drawing distance between the numbers-only and letters-only conditions. Response time was recorded for each condition.

\section{MRI acquisition}

Approximately 2 weeks after the behavioral testing session, participants returned for the scanning session. Diffusion and functional resting-state data were acquired on a 3 tesla Siemens Trio scanner with a 12-channel head coil. For DTI, two sets of whole-brain 30-direction diffusion weighted data were collected with the following parameters: $T R=9000 \mathrm{~s}$, $\mathrm{TE}=90 \mathrm{~ms}, b=900 \mathrm{~s} \cdot \mathrm{mm}^{-2}, 32$ oblique-axial slices with $5 \mathrm{~mm}$ thickness, FOV $=242 \mathrm{~mm}$. For the resting-state data, 30 gradient-EPI oblique axial slices with $5 \mathrm{~mm}$ thickness were obtained for the entire brain using a $\mathrm{T} 2{ }^{*}$-weighted pulse sequence during a 6 min and 20 second resting state. Participants were instructed to keep their heads still, keep their eyes open, and not fall asleep. After the resting-state scan, participants were asked whether they had complied with these instructions. The scanning parameters were TR $=2 \mathrm{~s}, \mathrm{TE}=30 \mathrm{~ms}, \mathrm{FOV}=200 \mathrm{~mm}, 64 \times$ 64 matrix. We also collected 1601 -mm-thick oblique axial slices of 3D MPRAGE T1 images with TR $=2 \mathrm{~s}, \mathrm{TE}=2.63 \mathrm{~ms}, \mathrm{FOV}=256 \mathrm{~mm}$ to create a sample-specific anatomical image for registration and overlay.

\section{DTI data analysis}

We used FSL (version 4.1.4, Smith et al., 2004) to carry out preprocessing and statistical analyses. Diffusion images were corrected for eddy-current distortion and head motion. B-vectors were rotated according to the log file generated from the eddy-current correction procedure. Subsequently, the no-diffusion images were smoothed with a Gaussian kernel of $1 \mathrm{~mm} \sigma$ and concatenated with the diffusion-weighted images. A binary brain mask was extracted from the no-diffusion image for subsequent diffusion tensor fitting. The mask was overlaid onto the nodiffusion image to ensure all the brain matter was included in the mask. Missing voxels within the brain were manually filled out using the mask tool in FSLView. A tensor model was then fit to the corrected data that resulted in FA and eigenvalues for three orthogonal directions $\left(\lambda_{1}, \lambda_{2}\right.$, $\left.\lambda_{3}\right)$. The eigenvalue along the primary tract was referred to as $\operatorname{AD}\left(\lambda_{1}\right)$, while the average of the other two orthogonal eigenvalues was considered to reflect $\mathrm{RD}\left(\lambda_{2,3}\right)$. These preprocessing steps were repeated for each participant.

Group analysis followed the standard protocol of Tract-Based Spatial Statistics (TBSS, version 1.2, Smith et al., 2006) in FSL. A representative FA image was chosen from the entire sample, by matching each participant's image to the images from each of the other participants in the sample and identifying the image that had the smallest amount of warping across the whole sample (i.e., the most representative image). This procedure resulted in a sample-specific target image that was then affinetransformed to MNI-152 space. FA images of all other subjects were nonlinearly transformed to the target image and affine-aligned to the MNI-152 space in a combined process to avoid resampling the images twice. All participants' FA images were merged and averaged to create a single mean $4 \mathrm{D}$ image. This mean $4 \mathrm{D}$ image was entered in the FA skeletonization program to generate a skeleton that only included voxels that were identified as white matter across all the subjects in the study. Next, we computed permutation-based voxelwise statistics on the skeletonized FA data examining two one-tailed comparisons, i.e., whether bilinguals had higher FA values than monolinguals and vice versa (randomize in FSL, v. 2.1, Nichols and Holmes, 2002). Results were corrected for wholebrain comparisons and were thresholded at $p<0.05$. 


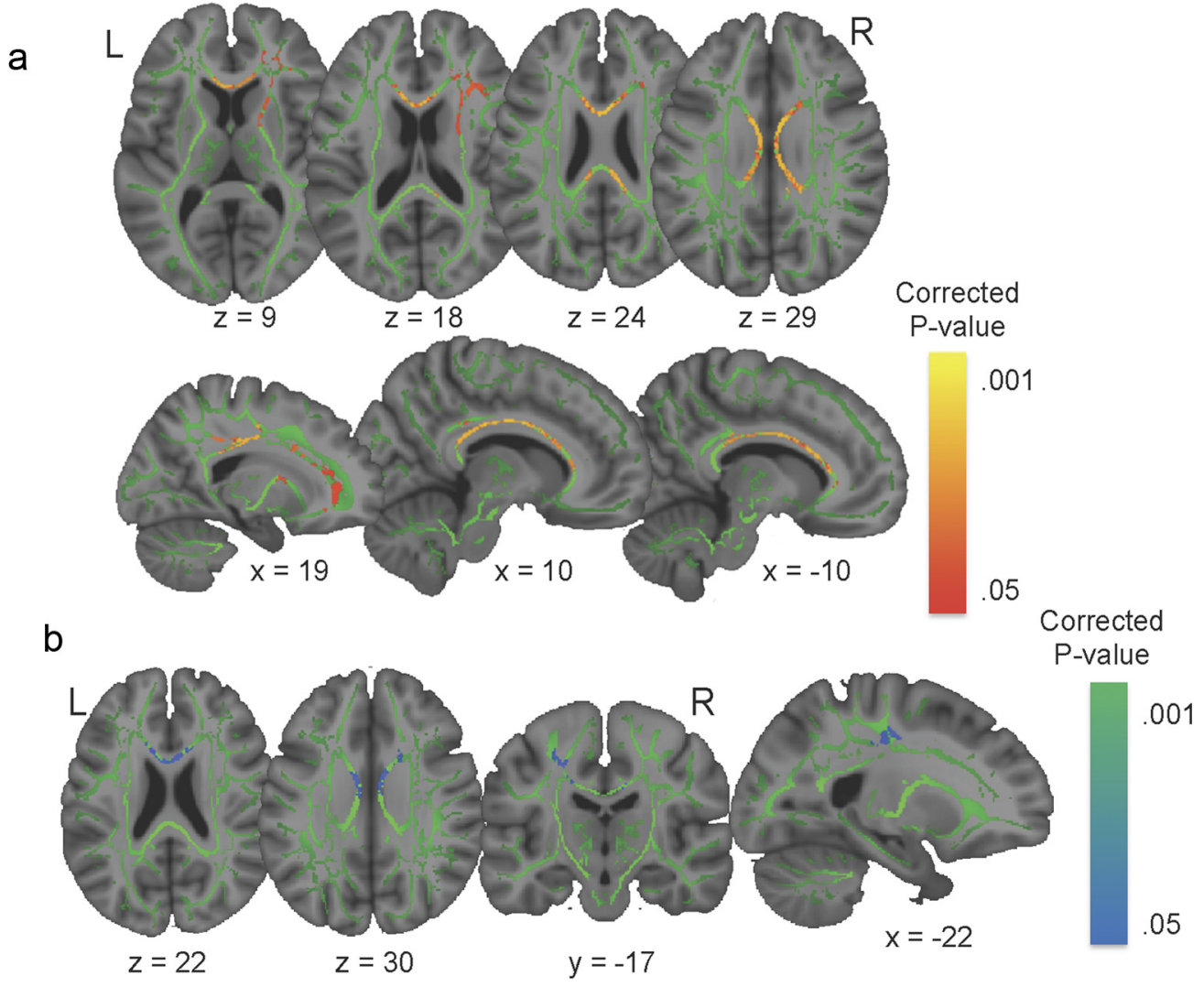

Figure 1. Group differences in FA and RD. $\boldsymbol{a}$, Increased FA in bilinguals compared to monolinguals. Colored voxels (orange-red) are significant clusters ( $p<0.05$, corrected) in corpus callosum, extending to bilateral superior longitudinal fasciculi and right inferior frontal-occipital fasciculus, shown on a white matter skeleton (green) and overlaid onto the MNI- $152 \mathrm{~T} 1$ brain (seen in gray). $\boldsymbol{b}$, Increased RD in monolinguals relative to bilinguals. Colored voxels (blue) are significant clusters ( $p<0.05$, corrected) overlapping with part of the WM tracts identified in $\boldsymbol{a}$ ).

\section{Resting-state functional data analysis}

The first 10 TRs of the resting-state functional data were excluded from the analysis to avoid signal instability. Subsequent data were corrected for slice timing, motion artifacts, and physiological signal and spatially normalized to standard Montreal Neurological Institute (MNI) space using the 152-subject template. White matter signal was partialled out as nuisance variable using regression. The normalized images were smoothed with an $8 \mathrm{~mm}$ FWHM resulting in isotropic voxel size of $4 \mathrm{~mm}$ for subsequent analysis. These preprocessing steps were performed using Analysis of Functional Neuro-Images (AFNI) (Cox and Hyde, 1997). All the coordinates reported subsequently are in MNI space.

The preprocessed resting-state data were analyzed with seed partial least squares (PLS; McIntosh et al., 1996, 2004; for a recent review, see Krishnan et al., 2011) following the procedures reported by Grigg and Grady (2010) for resting-state functional connectivity analysis. Seed PLS is a data-driven multivariate statistical technique that reveals functional activity across the entire brain that correlates with activity in a seed voxel (or region) chosen a priori. The covariance between activity in the seed and other brain voxels is decomposed into latent variables (LVs) that can identify multiple patterns of functional connectivity. The advantage of using PLS is the consideration of the entire resting-state (resampled to 34 blocks of 5 consecutive volumes) simultaneously, thereby reflecting both the temporal and spatial characteristics of intrinsic brain activity at rest. Furthermore, the decomposition and associated resampling techniques consider all time points and voxels simultaneously, thus avoiding the problem of multicollinearity and post hoc multiple correction of the $p$ values. Because of its ability to identify groups of brain regions with covarying functional connectivity, this technique is methodologically ideal for the investigation of large-scale brain networks (McIntosh, 2000). Activity in each seed voxel (see Results for seed location) was extracted and correlated (across participants) with all other brain voxels for each of the 34 time blocks in the resting run; PLS then was used to identify patterns of correlation that differed between bilinguals and monolinguals. Significance of the LVs was determined by 1000 permutation tests, using resampling without replacement. Robustness of each voxel's contribution to a LV was provided by a bootstrap that resampled the data 300 times (with replacement) to estimate the standard error of the weight of each voxel on the LV. A bootstrap ratio, calculated as the ratio of each weight to its standard error, was thresholded at \pm 3 , equivalent to $p \leq 0.0027$. Significant clusters were further thresholded to include at least 10 voxels.

\section{Results}

Consistent with our hypothesis of better maintenance of white matter integrity in bilinguals, higher FA values in the CC $(p<$ 0.05 , corrected, Fig. $1 a$ ) were seen in the bilingual group, extending posteriorly to the bilateral superior longitudinal fasciculi, and anteriorly to the right inferior frontal-occipital fasciculus and uncinate fasciculus. Monolinguals did not show higher FA values than bilinguals in any voxel. TBSS was also used to assess betweengroup differences in axial and radial diffusivity. There was no group difference in $\mathrm{AD}$, but monolinguals had higher $\mathrm{RD}$ in the body of the CC, overlapping with some of the areas where bilinguals had higher FA $(p<0.05$, corrected, Fig. $1 b)$. Note that the FA values in the genu of CC for both groups (bilinguals: $M=$ $0.47, \mathrm{SD}=0.04$; monolinguals: $M=0.44, \mathrm{SD}=0.03$ ) were lower than those usually reported for younger adults $(M=0.55, \mathrm{SD}=$ 0.04 from Davis et al., 2009), as would be expected.

To test the hypothesis that greater WM integrity in the bilinguals would be associated with a more distributed pattern of functional activity, we assessed functional connectivity of two seed voxels. We chose seeds in bilateral inferior frontal gyri because these regions are important for bilingual language switching (Garbin et al., 2010; Luk et al., 2011), and we found greater FA 
a

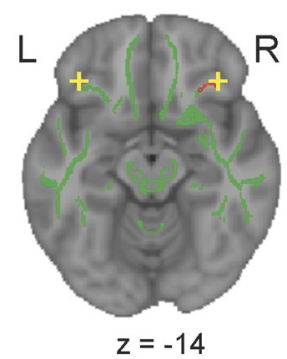

b

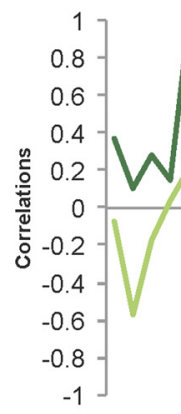

-Monolingual —Bilingual
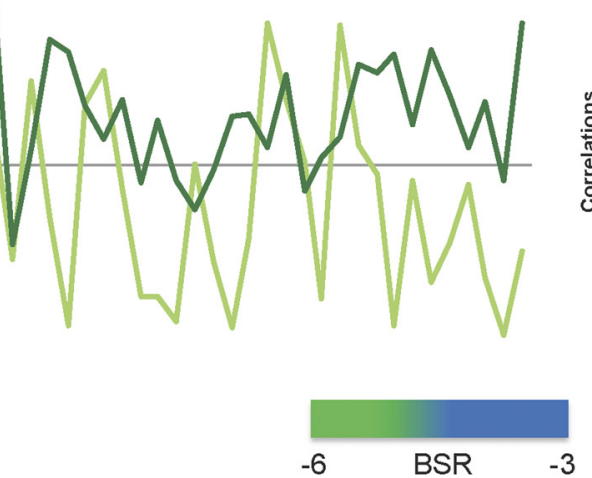

d

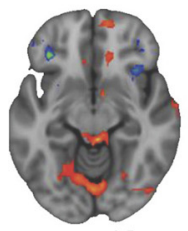

$z=-12$
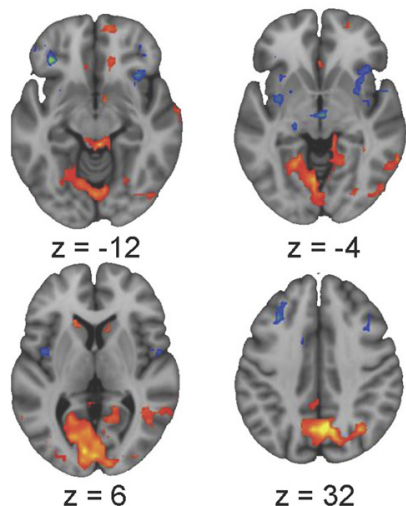

$z=-4$

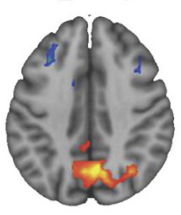

$z=32$
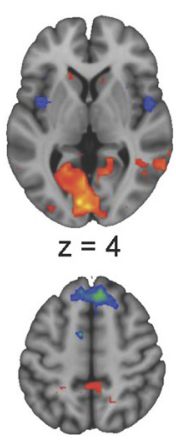

$z=52$
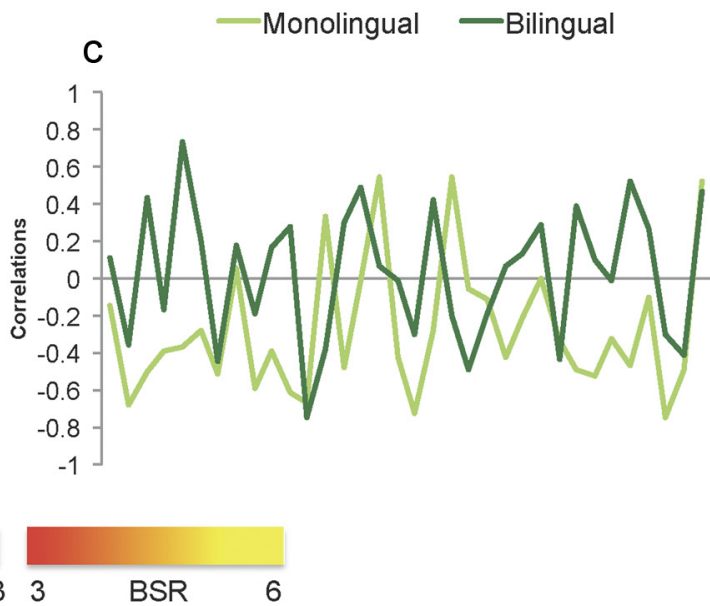

e

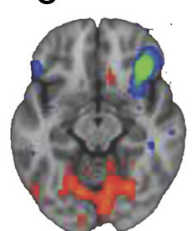

$z=-12$

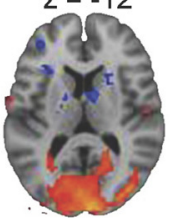

$z=12$

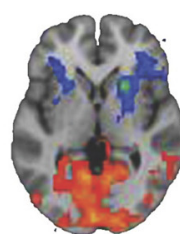

$\mathbf{z}=0$

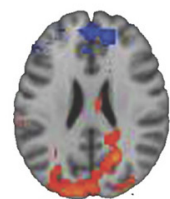

$z=24$

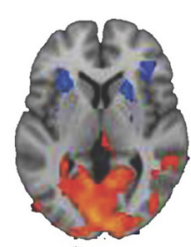

$z=4$

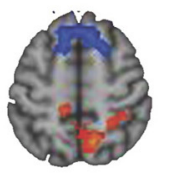

$z=52$

Figure 2. Resting-state functional connectivity for monolinguals and bilinguals. $\boldsymbol{a}$, Locations of the two seed voxels in LIFG and RIFG. $\boldsymbol{b}, \boldsymbol{c}$, Correlations between activity in LIFG and RIFG seed voxel and activity in the positive and negative brain networks across a 6 min resting state, respectively. $\boldsymbol{d}, \boldsymbol{e}$, Axial brain images showing bootstrap ratios greater than \pm 3 . Warm-colored voxels indicate that positively correlating activity was observed in the frontal-posterior network, which was strongly represented in bilinguals with the LIFG seeds in $\boldsymbol{d}$. Cool-colored voxels indicate negatively correlating activity in the anterior left-right network, similar for both monolinguals and bilinguals in both IFG seeds.

in the right inferior frontal gyrus, as well as in the CC connecting the frontal lobes, in bilinguals (see Fig. 1). The right inferior frontal seed (MNI: $x=38, y=31, z=-14$ ) was located in gray matter adjacent to where we observed a group difference in FA (see Fig. 2a). The left seed was located in the homologous region of the inferior frontal gyrus $(x=-38, y=31, z=-14$; Fig. $2 a)$. Although no FA difference was observed here, this seed was very similar to a region identified in a meta-analysis of brain regions active during bilingual switching (Luk et al., 2011, $x=-33, y=$ $21, z=-14)$.

For the left inferior frontal seed, we found a statistically reliable pattern that differentiated bilinguals from monolinguals (accounting for $6 \%$ of the variance in the data, $p<0.001$ ). Positive correlation values in Figure $2 b$ indicate a positive correlation between seed activity and activity in the warm-colored regions seen in Figure $2 d$; a negative correlation indicates the opposite pattern, i.e., that seed activity was positively correlated with activity in the cool-colored regions. These correlation distributions were significantly different between the groups (Wilcoxon normal approximation $Z=-4.2, p<0.0001$ ). Thus, for the left inferior frontal gyrus, bilinguals had stronger functional connectivity with bilateral middle temporal gyri, right inferior parietal lobule, precuneus, bilateral middle occipital gyri, and left caudate. This increase in connectivity with posterior regions suggests more long-range frontal-occipital and frontal-parietal functional connections in the bilinguals. In contrast, the monolinguals showed stronger functional connectivity between the left inferior frontal region and other anterior areas, including bilateral precentral gyri, right superior frontal gyrus, right middle frontal gyrus, and left medial frontal gyrus, suggesting stronger functional connec- 
tions within the frontal lobes and closer neighbors (see Notes for more information). This pattern of stronger functional connectivity between anterior-posterior regions in bilinguals and stronger intrafrontal connectivity in monolinguals is consistent with the higher FA values seen in long-range WM connections in the bilinguals, particularly in the longitudinal fasciculi.

The right inferior frontal gyrus seed also showed a statistically significant functional connectivity pattern that differentiated the two language groups ( $p<0.001$, accounting for $4.4 \%$ of the data variance). The correlations in the groups across the resting state run were less distinct than seen for the left inferior frontal region (see Fig. $2 c$ ), but were nevertheless significantly different (Wilcoxon normal approximation $Z=3.4, p<0.0008$ ). Interestingly, the pattern of group differences was similar to that seen for the left inferior frontal region (see Notes for more information). Monolinguals had stronger functional connectivity between the right inferior frontal seed and other frontal regions, whereas bilinguals had stronger functional interactions between the seed and occipitoparietal regions (Fig. 2e).

\section{Discussion}

Successful management of two languages on a daily basis requires an effective cognitive mechanism to manage attention to two active languages (Green, 1998). Constant recruitment of this mechanism by bilinguals leads to better behavioral performance on executive function tasks that measure cognitive control for both verbal and nonverbal stimuli (Costa et al., 2008; Blumenfeld and Marian, 2011). The present study shows that this prolonged bilingual experience leads to enhanced WM connectivity, which may be one mechanism underlying the bilingual advantage observed in EF performance. This finding is consistent with the idea that enriched experience contributes to brain reserve by protecting WM against age-related deterioration (Petrosini et al., 2009). In the present study, bilingualism was related to maintenance of WM integrity in older adults, primarily as a result of less radial diffusivity, implying that lifelong experience with enhanced cognitive control influences brain structure. Furthermore, bilinguals showed more distributed resting-state functional connectivity in frontal regions close to where the WM structural difference was observed, indicating a functional correlate of the WM difference.

The monolingual and bilingual older adults in our study had highly comparable demographic backgrounds and neuropsychological performance on standardized tests. The group differences observed in WM integrity and in resting-state functional connectivity indicate distinct neural patterns even when behavior was held constant. In a recent study, Schweizer et al. (2011) have shown that bilingual patients diagnosed with probable Alzheimer's disease showed a greater amount of brain atrophy compared to their monolingual peers, matched on cognitive performance and education level. An interesting observation in that study was that the bilinguals achieved comparable cognitive performance despite their greater brain atrophy. Our results showing better WM integrity in healthy older bilinguals, together with the data from demented patients, indicate WM could provide a "reserve" factor, to compensate for deteriorating gray matter, in turn sustaining cognitive performance.

Our results demonstrate a relationship between lifelong bilingual experience, maintained WM integrity, and more distributed functional connectivity in older adults. This finding is parallel to animal research showing that an enriched environment enhances adult neurogenesis (Beauquis et al., 2010). Although the biological mechanism for these structural changes in response to life experience is not known, the present results provide the first evidence that a naturally occurring life experience is related to WM plasticity in humans. We suggest that the enhanced structural and functional connectivity in bilingual older adults may provide at least part of the neural basis for the phenomenon of "brain reserve."

\section{Notes}

Please refer to the following link for supplemental information on participants' demographic information and brain coordinates for the resting-state analysis: http://gseacademic.harvard.edu/ lukgi/JoN_ 2011_SI_Tables.pdf. This material has not been peer reviewed.

\section{References}

Beaulieu C (2009) The biological basis of diffusion anisotropy. In: Diffusion MRI: from quantitative measurement to in vivo neuroanatomy. Diffusion MRI (Johansen-Berg H, Behrens TEJ, eds), pp 105-126. London, UK: Elsevier.

Beauquis J, Roig P, De Nicola AF, Saravia F (2010) Short-term environmental enrichment enhances adult neurogenesis, vascular network and dendritic complexity in the hippocampus of type 1 diabetic mice. PLoS ONE 5:e13993.

Bialystok E, Craik FIM (2010) Cognitive and linguistic processing in the bilingual mind. Curr Dir Psychol Sci 19:19-23.

Bialystok E, Craik F, Luk G (2008) Cognitive control and lexical access in younger and older bilinguals. J Exp Psychol Learn Mem Cogn 34:859-873.

Blumenfeld HK, Marian V (2011) Bilingualism influences inhibitory control in auditory comprehension. Cognition 118:245-257.

Costa A, Hernández M, Sebastián-Gallés N (2008) Bilingualism aids conflict resolution: evidence from the ANT task. Cognition 106:59-86.

Cox RW, Hyde JS (1997) Software tools for analysis and visualization of fMRI data. NMR Biomed 10:171-178.

Davis SW, Dennis NA, Buchler NG, White LE, Madden DJ, Cabeza R (2009) Assessing the effects of age on long white matter tracts using diffusion tensor tractography. Neuroimage 46:530-541.

Delis DC, Kaplan E, Kramer JH (2001) Design Fluency subtest from the Delis-Kaplan Executive Function System (D-KEFS). San Antonio, TX: The Psychological Corporation.

Fjell AM, Walhovd KB (2010) Structural brain changes in aging - courses, causes and cognitive consequences. Rev Neurosci 21:187-221.

Folstein MF, Folstein SE, McHugh PR (1975) Mini mental state: a practical method for grading the cognitive state of patients for the clinician. J Psychiatr Res 12:189-198.

Garbin G, Sanjuan A, Forn C, Bustamante JC, Rodriguez-Pujadas A, Belloch V, Hernandez M, Costa A, Avila C (2010) Bridging language and attention: brain basis of the impact of bilingualism on cognitive control. Neuroimage 53:1272-1278.

Green DW (1998) Mental control of the bilingual lexico-semantic system. Biling Lang Cogn 1:67-81.

Grigg O, Grady CL (2010) Task-related effects on the temporal and spatial dynamics of resting-state functional connectivity in the default network. PLoS ONE 5:e13311.

Krishnan A, Williams LJ, McIntosh AR, Abdi H (2011) Partial least squares (PLS) methods for neuroimaging: a tutorial and review. Neuroimage 56: 455-475.

Luk G, Anderson JA, Craik FI, Grady C, Bialystok E (2010) Distinct neural correlates for two types of inhibition in bilinguals: response inhibition versus interference suppression. Brain Cogn 74:347-357.

Luk G, Green DW, Abutalebi J, Grady CL (2011) Cognitive control for language switching in bilinguals: a quantitative meta-analysis on functional neuroimaging studies. Lang Cogn Process, in press.

Madden DJ, Spaniol J, Costello MC, Bucur B, White LE, Cabeza R, Davis SW, Dennis NA, Provenzale JM, Huettel SA (2009) Cerebral white matter integrity mediates adult age differences in cognitive performance. J Cogn Neurosci 21:289-302.

McIntosh AR (2000) Towards a network theory of cognition. Neural Netw 13:861-870.

McIntosh AR, Bookstein FL, Haxby JV, Grady CL (1996) Spatial pattern analysis of functional brain images using partial least squares. Neuroimage 3:143-157.

McIntosh AR, Lobaugh NJ (2004) Partial least squares analysis of neuroimaging data: applications and advances. Neuroimage 23:S250-S263. 
Moreno S, Bialystok E, Wodniecka Z, Alain C (2010) Conflict resolution in sentence processing by bilinguals. J Neurolinguistics 23:564-579.

Nichols TE, Holmes AP (2002) Nonparametric permutation tests for functional neuroimaging: a primer with examples. Hum Brain Mapp 15:1-25.

Petrosini L, De Bartolo P, Foti F, Gelfo F, Cutuli D, Leggio MG, Mandolesi L (2009) On whether the environmental enrichment may provide cognitive and brain reserves. Brain Res Rev 61:221-239.

Reitan RM (1958) The validity of the Trail Making Test as an indicator of organic brain damage. Percept Mot Skills 8:271-276.

Schweizer TA, Ware J, Fischer CE, Craik FI, Bialystok E (2011) Bilingualism as a contributor to cognitive reserve: evidence from brain atrophy in Alzheimer's disease. Cortex. Advance online publication. doi:10.1016/j.cortex.2011.04.009.
Smith SM, Jenkinson M, Woolrich MW, Beckman CF, Behrens TEJ, Johansen-Berg H, Bannister PR, De Luca M, Drobnjak I, Flitney DE, Niazy RK, Saunders J, Vickers J, Zhang Y, De Stefano N, Brady JM, Matthews PM, et al. (2004) Advances in functional and structural MR image analysis and implementation as FSL. Neuroimage 23:208-219.

Smith SM, Jenkinson M, Johansen-Berg H, Rueckert D, Nichols TE, Mackay CE, Watkins KE, Ciccarelli O, Cader MZ, Matthews PM, Behrens TE, et al (2006) Tract-based spatial statistics: voxelwise analysis of multi-subject diffusion data. Neuroimage 31:1487-1505.

Stroop JR (1935) Studies of interference in serial verbal reactions. J Exp Psychol 18:643-662.

Zachary RA (1986) Shipley Institute of Living Scale: Revised manual. Los Angeles: Western Psychological Services. 